\title{
DIE UNAUFLÖSLICHKEIT DER EHE NACH AMORIS LAETITIA: VERSUCH EINER THEOLOGISCHEN AUSEINANDERSETZUNG MIT BEDENKEN MANCHER POLNISCHER MORALTHEOLOGEN
}

K O N R A D G L O M B I K

\section{ABSTRACT \\ Indissolubility of Marriage according to Amoris Laetitia: \\ Theological Discussion of Doubts of Some Polish Moral Theologians}

The publication of Amoris laetitia initiated numerous discussions in the circles of theologians who focus on the question of the possibility of sacraments for remarried divorced couples. One of the fundamental theological questions in reference to marriage is the problem of the indissolubility. The apostolic exhortation shows no chapter or any point about the indissolubility of marriage but Amoris laetitia in numerous places talks about the problem of divorce and the stable indissoluble character of marriage. The article presents doubts of Polish moral theologians with regard to the indissolubility of marriage and attempts to explain and to answer them. The problem concerns two kinds of arguments. The first one is the language and ideas used in Amoris laetitia for presenting the indissolubility of marriage. The second line concerns the impaired indissolubility of marriage, which for the Polish moral theologians is the possibility of sacraments for remarried divorced people.

Key words:

Indissolubility of marriage; Theology of marriage; Sacrament of marriage; Amoris laetitia; Polish moral theology.

DOI: $10.14712 / 23363398.2020 .46$

$\mathrm{D}$ as apostolische Schreiben Amoris laetitia von Papst Franziskus (weiter nur AL) löste in den Kreisen der Theologen zahlreiche Diskussionen aus, die sich auf das achte Kapitel und die Frage der Zulassung der wiederverheirateten geschiedenen Katholiken zu den 
Sakramenten konzentrierten. Dieses Papstschreiben beinhaltet jedoch auch andere Gedanken zum modernen personalistischen Verständnis der Ehe und versucht, pastorale Lösungen für die mehrschichtigen Probleme und Krisen des ehelichen und familiären Lebens anzubieten. Eine der grundsätzlichen theologischen Fragen angesichts der Ehe ist das Problem ihrer Unauflöslichkeit, die auf dem Wort Jesu gründet und in der Tradition mit ihrer Sakramentalität eng verknüpft war. AL entstand als Ergebnis von zwei Bischofssynoden zu Ehe und Familie im Herbst 2014 und 2015, die u.a. auch das Problem der Scheidungen katholischer Ehen in der Gegenwart besprochen haben. Auch wenn in AL weder ein Kapitel noch ein Punkt der Unauflöslichkeit der Ehe gewidmet ist, wird hier über das Problem der Ehescheidungen und den dauerhaften und unauflöslichen Charakter der sakramentalen Ehe gesprochen.

AL wurde Gegenstand zahlreicher Diskussionen und Studien unter polnischen Moraltheologen. ${ }^{1}$ Auch wenn darin kaum unmittelbar die Unauflöslichkeit der Ehe reflektiert wurde, gibt es einzelne Beiträge, die diese Frage nach der Unauflöslichkeit der Ehe auf dem Hintergrund anderer Fragen, überwiegend der Zulassung der wiederverheirateten Geschiedenen zu den Sakramenten, indirekt ansprechen. Absicht des vorliegenden Beitrags ist es, die Bedenken und Fragen ausgewählter polnischer Theologen bezüglich der Unauflöslichkeit der Ehe darzustellen und sich mit ihnen kritisch auseinanderzusetzen. Die Frage nach der Unauflöslichkeit in Diskussionen zu AL scheint aus zwei Argumentationslinien zu bestehen. Die erste betrifft die Sprache und Begriffe, mittels deren in AL der unauflösliche Charakter der Ehe dargestellt und begründet wurde. Die zweite Argumentationslinie ist mit der Hinterfragung des Grundsatzes der Unauflöslichkeit der

1 Seit dem Erscheinen von AL gab es in Polen mehrere Konferenzen, an denen auch Moraltheologen teilgenommen haben. Mehr zur Rezeption der Exhortation bei den polnischen Moraltheologen in: Ireneusz Mroczkowski, ,Polska recepcja adhortacji Amoris laetitia papieża Franciszka‘, Społeczeństwo 27, Nr. 4 (2017): 78-94. Das apostolische Schreiben wurde auch das Thema der Jahrestagung der polnischen Moraltheologen, die vom 11. bis 13. Juni 2017 in Nysa stattgefunden hat. Bei dieser Konferenz gab es heftige Diskussionen, angeregt von zwei Vortragenden (Eberhard Schockenhoff und Livio Melina), die gegensätzliche Positionen zu AL vertraten. Die Mehrheit der polnischen Moraltheologen mit wenigen Ausnahmen teilte die gegen AL skeptische Position von Melina. - Vgl. dazu Janusz Podzielny, ,Sprawozdanie z ogólnopolskiej konferencji naukowej „Implikacje teologicznomoralne adhortacji Amoris laetitia”, Nysa, 11-13.06.2017 r., Studia Teologiczno-Historyczne Ślaska Opolskiego, 37, Nr. 2 (2017): 255-258. 
Ehe verbunden, die laut mancher polnischer Moraltheologen in der Zulassung zu den Sakramenten der wiederverheirateten geschiedenen Katholiken besteht.

\section{Das Problem der Begriffe und der Sprache}

Über die Unauflöslichkeit der Ehe ist in AL im dritten Kapitel von Berufung der Familie und vom göttlichen Plan zur Ehe die Rede. Der Papst zitiert die Relatio Synodi aus dem Jahre 2014 und das Wort Jesu „Was Gott verbunden hat, das darf der Mensch nicht trennen“ (Mt 19,6) und stellt fest, dass die Unauflöslichkeit der Ehe nicht als „Joch“, sondern als „Gabe“ für die in der Ehe verbundenen Menschen zu verstehen sei.

Auf diese Weise zeigt Jesus, wie Gottes Entgegenkommen den Weg der Menschen immer begleitet, die verhärteten Herzen mit seiner Gnade heilt und verwandelt und sie über den Weg des Kreuzes auf ihren Ursprung hin ausrichtet. Aus den Evangelien geht klar das Beispiel Jesu hervor (AL 62).

Nach dem polnischen Moraltheologen Ireneusz Mroczkowski wird in der Sicht der Unauflöslichkeit der Ehe in AL zwischen Pflicht und Ansporn unterschieden. Daher kommt die Frage, ob die Unauflöslichkeit der Ehe mit der Verpflichtung bis zum Eheversprechen hin geschützt werden soll oder lieber auf der Ebene einer Gabe bleiben soll, die, auch wenn sie abgelehnt wird, nicht als Ehebruch gelten darf. Es ist kein Zufall, so Mroczkowski, dass Theologen, die das achte Kapitel von AL „liberal“ auslegen, das heißt aus seiner Sicht die Zulassung der wiederverheirateten Geschiedenen zu den Sakramenten der Versöhnung und der Eucharistie befürworten, das Versprechen der ehelichen Treue und die Sünde des Ehebruchs in ihren Beiträgen nicht erwähnen. ${ }^{2}$

Diese Argumente müssen aber kritisch hinterfragt werden: Zum einen nehmen Mroczkowskis Aussagen zu Treue und Ehebruch nicht völlig wahr, was AL im Leben der wiederverheirateten Geschiedenen würdigt, nämlich moralische Werte wie gefestigte Verbindung,

2 Vgl. Ireneusz Mroczkowski, Etos miłosierdzia a wierność małżeńska: moralne dylematy osób żyjących w związkach niesakramentalnych w świetle adhortacji Amoris laetitia papieża Franciszka (Płock: Płocki Instytut Wydawniczy, 2017), 28. 
erwiesene Treue, großherzige Hingabe, christliches Engagement (vgl. AL 298). Zum anderen ist es angebracht, angesichts der Vielschichtigkeit der Problematik über den Begriff des Ehebruchs nachzudenken und ihn differenzierter zu verstehen, worauf Eberhard Schockenhoff hingewiesen hat:

Es stellt sich die Frage, ob das Leben in einer zivilen Zweitehe, in der moralische Werte wie Liebe und Treue, Verlässlichkeit und Füreinander-Eintreten gelebt werden, überhaupt als Ehebruch bezeichnet werden kann, wenn die erste Ehe endgültig gescheitert und nach menschlichem Ermessen eine gemeinsame Eheführung der seitdem getrennt lebenden Gatten nicht mehr möglich ist. Wenn die erste Ehe nur noch als Rechtsfiktion oder als geistlicher Torso, aber nicht mehr als gelebte Realität existiert, kann das Zusammenleben mit einem anderen Partner in einer zivilen Zweitehe nicht als fortgesetzter Ehebruch qualifiziert werden. Dies ist zumindest dann unmöglich, wenn man die Ehe nicht mehr als einen Vertrag zum Zwecke der Übereignung der Geschlechtseigenschaften an den Partner, sondern als eine personale Lebensgemeinschaft und ein ganzheitliches Sich-Schenken der Ehegatten versteht, wie es der Ehelehre des Zweiten Vatikanischen Konzils entspricht. Unter der Voraussetzung, dass die Ehe als ein „Bund“ und eine personal-ganzheitliche Lebensgemeinschaft verstanden wird, erweist sich die Annahme eines Ehebruchs gegenüber dem ersten Partner als absurd. Wenn die früheren Partner nicht mehr durch einen ganzheitlichen Lebensvollzug verbunden sind, haben sie auch kein Recht auf sexuelle Gemeinschaft mehr, das durch die in einer Zweitehe gelebte Sexualität verletzt werden könnte. ${ }^{3}$

Interessante Erläuterungen zur Sprache und zu den Begriffen über die Unauflöslichkeit der Ehe in AL stellen die Überlegungen von Kardinal Walter Kasper dar. Für ihn ist es klar, dass die kirchliche Lehre von der Unauflöslichkeit der Ehe im Zusammenhang mit der Botschaft Jesu vom Kommen des Reiches Gottes und seiner Barmherzigkeit zu deuten ist. Nach dem Wort Jesu „Was Gott verbunden hat, das darf der Mensch nicht trennen“ (Mt 19,6) soll die Unauflöslichkeit nicht als ein der Ehe auferlegtes Joch missverstanden werden. Das Eheband

3 Eberhard Schockenhoff, ,Traditionsbruch oder notwendige Weiterbildung? Zwei Lesarten des Nachsynodalen Schreibens „Amoris laetitia“", Stimmen der Zeit 142, Nr. 3 (2017): 147-158, hier 154 . 
(vinculum) darf man nicht wörtlich als Kette oder Fessel deuten. Das Eheband ist ein Geschenk im Sinne einer Zusage, dass Gott das Ja-Wort der Eheleute durch sein Ja trägt, die Eheleute auf ihrem Weg begleitet, die verhärteten Herzen heilt und auf dem Weg des Kreuzes auf das Leben in der österlichen Freude ausrichtet. ${ }^{4}$

Kasper weist auch darauf hin, dass der Begriff Unauflöslichkeit den Geschenkcharakter nur unvollkommen zum Ausdruck bringt, weil er aus dem Vertragsrecht, wonach gewisse Verträge bleibende Bindung begründen und somit unauflöslich sind, stammt. Mit diesem juristischen Begriff lässt sich das von Gottes Bundeszusage getragene gegenseitige Sich-Schenken der Eheleute nicht ausreichend zum Ausdruck bringen. Darum verwendet AL an vielen Stellen ein anderes biblisches und mehr der menschlichen Erfahrung entsprechendes Wort, nämlich „Treue“. Im ehelichen Treueversprechen verspricht Gott den Eheleuten seine Treue, die die fragile menschliche Treue trägt, umfasst, stärkt und vollendet. Auf diese Weise gelingt es Franziskus, die kirchliche Lehre neu zum Leuchten zu bringen. ${ }^{5}$

Papst Franziskus kritisiert in AL den Gebrauch der Sprache und Begriffe in der Darstellung der Ehe und Familie in der Verkündigung. Er nennt dabei vor allem die übertriebene Idealisierung und stellt fest:

Andere Male haben wir ein allzu abstraktes theologisches Ideal der Ehe vorgestellt, das fast künstlich konstruiert und weit von der konkreten Situation und den tatsächlichen Möglichkeiten der realen Familien entfernt ist. Diese übertriebene Idealisierung, vor allem, wenn wir nicht das Vertrauen auf die Gnade wachgerufen haben, hat die Ehe nicht erstrebenswerter und attraktiver gemacht, sondern das völlige Gegenteil bewirkt (AL 36).

Laut Mroczkowski lässt nur ein völlig realistischer biblischer Blick die richtigen Verhältnisse zwischen Idee und Idealisierung bewahren. Das Ideal der Ehe soll bewahrt werden und die Idealisierung der Ehe, d.h. die abstrakte Darstellung der Ehe - fern von den konkreten Situationen und Möglichkeiten der Familien - ist abzulehnen. Die biblische Lehre von der Unauflöslichkeit der Ehe soll im Schlüssel der entsprechenden theologischen Anthropologie, der integral verfassten Absicht

4 Vgl. Walter Kasper, Die Botschaft von Amoris laetitia: ein freundlicher Disput (Freiburg im Br.: Herder, 2018), 55-56.

5 Vgl. ebd., 57. 
Gottes zum Menschen, interpretiert werden. Eine solche Sicht der Ehe findet man, so Mroczkowski, in der Lehre von Papst Johannes Paul II. Für das Verständnis der Unauflöslichkeit der Ehe ist die biblische Botschaft zur Erschaffung von Mann und Frau und ihre gegenseitige Bezogenheit $($ Gen 2,24) und die Worte Jesu zur Ehescheidung (Mt 19,3-8; Mk 10,2-9) grundlegend. Er unterscheidet dabei zwischen der ontologischen und der moralischen Dimension der Unauflöslichkeit der Ehe. Die ontologische Unauflöslichkeit der Ehe ist ein im Sakrament der Ehe verankertes Werk Gottes, das im Ehebund, der nie aufgelöst werden kann, zum Ausdruck kommt. Die Christen sollen nicht nur diesen Bund nicht auflösen, sondern sie können ihn nicht auflösen (vgl. Mt 19,6). Die ethischen Forderungen, verbunden mit der Unauflöslichkeit der Ehe, bestehen in der Überwindung der Begehrlichkeiten und ihrer Folgen in der Gestalt von Ehebruch. In diesem Sinne wird von der moralischen Unauflöslichkeit gesprochen, die den Ehebund, der fragil ist, schützt. Diese moralische Unauflöslichkeit der Ehe, die von der persönlichen Anstrengung und dem Eheversprechen unterstützt wird, ist ein Ideal, das von zahlreichen Ehepaaren nicht erreicht wird. Daher ist die Begleitung solcher Paare, aber nicht die Auflösung der Ehen begründet, so Mroczkowski. ${ }^{6}$

Es ist jedoch hinzuzufügen, dass hier dem Lehrschreiben unterstellt wird, was in AL nicht steht. Es muss betont werden, dass es in AL keine Stelle gibt, an der die Unauflöslichkeit der Ehe weder in Frage gestellt noch daran gezweifelt noch dieser Grundsatz gelockert wird. Papst Franziskus stellt nirgendwo fest, dass die Lehre zur Unauflöslichkeit der Ehe so geändert wird, dass man ein Eheband auflösen und ein anderes ohne Weiteres eingehen könne. Eine derartige Schlussfolgerung ist auch nicht aus der Ansicht zu ziehen, in der die wiederverheirateten Geschiedenen unter gewissen Voraussetzungen zu den Sakramenten zugelassen werden können. Diese Möglichkeit hat schon

${ }_{6}$ Vgl. Mroczkowski, Etos miłosierdzia a wierność matżeńska, 28-33; mehr zur Unterscheidung zwischen der moralischen und ontologischen Unauflöslichkeit der Ehe siehe auch: Marian Machinek, 'Die Diskussion über die Unauflöslichkeit der Ehe im Zusammenhang mit dem nachsynodalen apostolischen Schreiben Amoris laetitia: einige offene Fragen', Studia Teologiczno-Historyczne Ślaska Opolskiego 37, Nr. 1 (2017): 121-140, hier 126-127; Marian Machinek, 'Logika inkluzji papieża Franciszka: pytania otwarte na marginesie adhortacji Amoris laetitia', Forum Teologiczne 18 (2017): 197-211, hier 203-204. 
das apostolische Schreiben von Johannes Paul II. Familiaris consortio geöffnet. $^{7}$

Für die Probleme, die AL behandelt, nimmt die Aussage Jesu in der Bergpredigt eine Schlüsselrolle ein: „Wer seine Frau entlässt, obwohl kein Fall von Unzucht vorliegt, liefert sie dem Ehebruch aus; und wer eine Frau heiratet, die aus der Ehe entlassen worden ist, begeht Ehebruch“ (Mt 5,32). Der polnische Moraltheologe Marian Pokrywka zitiert diese Aussage ohne die Unzuchtklausel und bedauert, dass dieser Text in AL übersehen wurde. Er betont auch, dass sich Jesus in seiner Verkündigung einer konkreten normativen Sprache bedient und eindeutig Sünde benennt (vgl. Joh 8,11). Der Verzicht auf die normative Sprache in AL bewirkt laut Pokrywka, dass die Barmherzigkeit auf eine emotionale Empfindlichkeit herabgesetzt wird. Die Barmherzigkeit wird aber nur dann wahrgenommen, wenn sie auch die Wahrheit liebt, und nur die Wahrheit über die Sünde und die Barmherzigkeit kann den Menschen heilen. ${ }^{8}$

Laut Pokrywka ist die Kirchenlehre kein rigoroses System von Formeln. Ihre Ausdrucksformen dürfen semantisch entwickelt und verändert werden, ohne die grundsätzlichen Inhalte der ethischen Botschaft in Frage zu stellen. Wenn Jesus in der Bergpredigt den Ehebruch eindeutig als Sünde benennt, können wir diese Tat nicht als nur eine Unzulänglichkeit auf dem Weg zum Ideal, das nach dem individuellen Gewissen zu beurteilen ist, herabsetzen. Pokrywka unterstellt dem Papst, dass er in AL absichtlich die Aussagen von Veritatis splendor ausgelassen hat, in der der Universalismus und die Unveränderlichkeit der moralischen Gebote, besonders der in sich schlechten Handlungen, betont wurde und die abgelehnte kreative Interpretation des Gewissens, die zum doppelten Status der moralischen Wahrheit führt, verwendet. Daher darf die Sorge um die wiederverheirateten Geschiedenen und um die pastorale Bekehrung die Moraltheologie nicht dazu verleiten, auf den absoluten Charakter der moralischen Normen zu

\footnotetext{
Vgl. Eligiusz Piotrowski, ,Co dalej z Amoris laetitia w Polsce?‘, Znak, Nr. 10 (2019), In: https://bit.ly/3910fSO (22.6.2020).

8 Vgl. Marian Pokrywka, 'Teologicznomoralne implikacje adhortacji Amoris laetitia', in Towarzyszyć matżeństwu i rodzinie: inspiracje adhortacji apostolskiej Amoris laetitia dla duszpasterstwa rodzin, ed. Jacek Goleń (Lublin: Wydawnictwo KUL, 2017), 81-96, hier 90 .
} 
verzichten, so Pokrywka. Schließlich soll AL nur aus der Perspektive von Veritatis splendor ausgelegt werden. ${ }^{9}$

Die Logik von Pokrywka, in der das Auslassen der Aussagen von Veritatis splendor in AL als Absicht bzw. Infragestellung der universalen moralischen Normen verstanden wird, erscheint befremdlich. AL als ein pastorales Schreiben, hat weder zum Ziel die Inhalte anderer lehramtlicher Dokumente zu wiederholen noch die Lehre von den moralischen Normen und dem Gewissen erneuert zu vertiefen. Vielmehr sollen die Grundsätze der katholischen Moraltheologie in eine bessere und lebensnahe pastorale Sorge für die wiederverheirateten Geschiedenen eingeschlossen werden. AL ermutigt daher die Seelsorger, die Gläubigen in ihren irregulären Situationen zu begleiten, ihre Lebenssituationen zu unterscheiden und sie besser in die Gemeinschaft der Kirche einzugliedern. Diese pastorale Sorge, die AL kennzeichnet, stellt weder bisherige moraltheologische Grundsätze in Frage noch schafft sie neue kanonischen Vorschriften.

Robert Plich wirft AL vor, die Norm, die die Unauflöslichkeit der Ehe anordnet, und die Verbotsnorm des Ehebruchs mit dem Ideal zu verwechseln. Ideale haben, so Plich, eine andere Natur als Normen und Gebote. Ideale sind edle Angebote ohne Verpflichtungskraft und sind Gegenstand des Aufrufes und des Rates und nicht des Gebotes. Auf Ideale kann man hinweisen, zu ihrer Verwirklichung ermutigen, aber ihre Ausführung ist keine Pflicht und daher ihre Nichtbefolgung keine Sünde. Die Normen lehrt man, zu ihrer Befolgung erzieht man den Menschen und man fordert ihre Erfüllung, weil sie darauf hinweist, wie es sein soll und was die Pflicht ist. Laut Plich ist es merkwürdig, dass Papst Franziskus nicht zur Lehre und Forderung der Normen, sondern zur pastoralen Begleitung derer, die nicht imstande sind, die Ideale völlig zu verwirklichen, aufruft. Die Veränderung der Verbotsnormen zur Ehescheidung und zum Ehebruch mit den Idealen geht in AL mit der Veränderung des Verständnisses der Natur des moralischen Rechtes einher. Während Johannes Paul II. den verpflichtenden Charakter des Rechtes, der zur ständigen Überwindung der Schwierigkeiten treibt, betont, unterstreicht Franziskus eher die informative Natur des Rechtes, die weder treibt noch gebietet, sondern nur auf den Weg hinweist und ihn anbietet, behauptet Plich. Der informative und der verpflichtende Charakter des Rechtes müssen in keiner

9 Vgl. ebd., 94-95. 
Weise gegenseitig ausgeschlossen werden, sondern sich gegenseitig ergänzen und angenommen werden. Die Feststellung aus AL „Niemand darf auf ewig verurteilt werden, denn das ist nicht die Logik des Evangeliums!“ ist, laut Plich, mit der Lehre Jesu, der Apostel und der Kirche nicht zu vereinbaren. Denn wieso soll man den idealen Weg des moralischen Rechtes gehen, wenn man von vornherein weiß, dass andere nichtideale Wege keineswegs zur Verdammnis führen und man auf dem Weg auch andere Werte verwirklichen kann. ${ }^{10}$

Die Unauflöslichkeit ist für die Ehe kein Ideal, so weiter Plich, sondern eine Norm, von der der Wert und das Bestehen der Ehe abhängen. Daher hat die Sorge um die Unauflöslichkeit der Ehe und das Verbot ihrer Verletzung den Charakter der moralischen Pflicht und nicht nur die Bedeutung einer Aufforderung oder eines Ansporns. Weder das Verständnis für den Wert der Unauflöslichkeit der Ehe noch die Schwierigkeit ihrer Verwirklichung beeinflussen ihren normativen Charakter und sind kein Kriterium für die Unterscheidung zwischen Ideal und Norm. Die Unauflöslichkeit der Ehe ist eine Norm, die aufgrund der Unterstützung der Gnade im Sakrament der Ehe verwirklicht wird. Die Herabsetzung der absoluten Unauflöslichkeit der Ehe und des absoluten Verbots des Ehebruches auf die Ebene des außerpflichtmäßigen Ideals durch Franziskus, so Plich, bewirkt, dass auch das Gesetz der Gradualität in AL anders als in Familiaris consortio verstanden wird. Statt die eigenen Grenzen zu überwinden, um die völlige Verwirklichung der Moralnormen zu erreichen, wird bei Franziskus das Gesetz der Gradualität als Möglichkeit eines stufenweise zu verwirklichenden fakultativen Ideals dargestellt, das nicht erforderlich ist, sondern zu dem aufgerufen wird. ${ }^{11}$

Die oben dargestellte Kritik von Plich nimmt nicht genug wahr, dass AL von den objektiven Idealen spricht, die inmitten der Vielschichtigkeit und Begrenzungen in konkreten Situationen noch nicht verwirklicht sind (vgl. AL 303), und nicht von Idealen, die der Mensch freiwillig und beliebig außerpflichtmäßig in seinem Leben auswählt und befolgt. Der Verzicht auf die Sprache und Begriffe, die die moralischen Normen und Pflichten betonen, entspricht dem pastoralen Charakter des päpstlichen Schreibens, das als Ausgangspunkt die Erfahrung der

10 Vgl. Robert Plich, 'Legalistyczna neutralizacja nauki o czynach wewnętrznie złych i absolutnych zakazach moralnych w adhortacji Amoris laetitia', Teologia i Moralność 13, Nr. 1 (2018): 89-123, hier 107-109.

11 Vgl. ebd., 109. 
Menschen hat und die Absicht verfolgt, ihnen in den vielschichtigen Problemen zu helfen. Papst Franziskus erwähnt in seinem Schreiben an vierzehn Stellen das Wort „Unauflöslichkeit“, vermeidet dabei die biblischen Texte, die eine Möglichkeit der Schließung einer zweiten Ehe nahelegen würden und den Text Mt 19,3-9 in der Perspektive der Sünde erläutert. Auf diese Weise ist seine Stellung zur Unauflöslichkeit der Ehe indiskutabel. ${ }^{12}$

Die Bedenken von Pokrywka und Plich bezüglich der Sprache des päpstlichen Schreibens erläutern die Überlegungen von Walter Kasper. Mit Recht weist Kasper darauf hin, dass AL von einem neuen, frischen, befreienden Ton geprägt ist und nicht von einem abstrakten Familienbild spricht, sondern realistisch die Freuden und Schwierigkeiten der Ehe und Familien angeht. Es will weder kritisieren noch moralisieren noch indoktrinieren, sondern bedient sich einer offenen, unverkrampften und ansprechenden Sprache und drückt das Verständnis und die Wertschätzung für das Gute aus, das sich auch in Situationen finden kann, die der kirchlichen Lehre und Ordnung nicht oder nicht völlig entsprechen. Dieser Sprache folgt eine pastorale Konzeption, für die nicht der erhobene Zeigefinger, sondern die ausgestreckte helfende Hand charakteristisch ist. Hinhören, wertschätzen, begleiten, integrieren sind maßgebend. Hinter diesem pastoralen Ton steckt eine durchdachte theologische Position und eine dynamische Sicht des Menschund Christseins, für die das biblische Weg-Motiv maßgebend ist. Dabei gilt das Gesetz der Schritte, was keine nur schrittweise Gültigkeit des Gesetzes bedeutet. Das Gesetz gilt immer und ist kein fernes Ideal, sondern orientiert jeden einzelnen Schritt auf das Ziel hin. Meistens können Menschen nicht das Optimum, sondern nur das in ihrer Situation Bestmögliche tun und oftmals das kleinere Übel wählen. Im Leben gibt es nicht nur Schwarz und Weiß, sondern sehr unterschiedliche Nuancen und Schattierungen. ${ }^{13}$

Es ist auch Rocco Buttiglione zuzustimmen, der der Meinung ist, dass Papst Franziskus, wenn er über die Ehe als Ideal schreibt, in keiner Weise ihren normativen Charakter ausschließt. Die Überwindung

12 Vgl. Marek Dzik, 'Biblijny obraz rodziny i małżeństwa w adhortacji Amoris laetitia', in Towarzyszyć małżeństwu i rodzinie: inspiracje adhortacji apostolskiej Amoris laetitia dla duszpasterstwa rodzin, ed. Jacek Goleń (Lublin: Wydawnictwo KUL, 2017), 51-64, hier 61-62.

13 Vgl. Walter Kasper, 'Amoris laetitia: Bruch oder Aufbruch?,' Stimmen der Zeit 141, Nr. 11 (2016): 723-732, hier 724-725. 
einer bestimmten Verrechtlichung bedeutet noch lange nicht, die Bedeutung des Rechtes für das Leben der Kirche und der Gläubigen in Frage zu stellen. Die Unauflöslichkeit der Ehe ist in AL eindeutig bestätigt und der normative Charakter der Worte Jesu wird in ihr völlig anerkannt (vgl. AL 61-62). Die Unauflöslichkeit der Ehe wurde nicht zum Ideal im Sinne eines evangelischen Rates herabgesetzt. Ein Gläubiger, der in einer Ehe lebt, begeht keine Sünde wegen Nichtbefolgung des evangelischen Keuschheitsrates, sondern begeht eine Todsünde im Fall des Ehebruches. Es gibt in AL keine Stelle, die die bisherige kirchliche Position zu den wiederverheirateten Geschiedenen, die in der Situation einer ernsthaften Unordnung leben, in Frage stellt. Diese Frage wird jedoch aus der Sicht der mildernden Umstände behandelt, was den Verstoß gegen die Norm voraussetzt. ${ }^{14}$

\section{Zum Problem der Kohärenz zwischen der Eucharistie und dem Ehesakrament}

Das Thema der Kohärenz zwischen der Eucharistie und dem Sakrament der Ehe beschreibt Papst Franziskus wie folgt:

Die christliche Ehe, ein Abglanz der Vereinigung Christi und seiner Kirche, wird voll verwirklicht in der Vereinigung zwischen einem Mann und einer Frau, die sich in ausschließlicher Liebe und freier Treue einander schenken, einander gehören bis zum Tod, sich öffnen für die Weitergabe des Lebens und geheiligt sind durch das Sakrament. Dieses Sakrament schenkt ihnen die Gnade, um eine Hauskirche zu bilden und ein Ferment neuen Lebens zu sein. Andere Formen der Vereinigung widersprechen diesem Ideal von Grund auf, doch manche verwirklichen es zumindest teilweise und analog. Die Synodenväter haben betont, dass die Kirche nicht unterlässt, die konstruktiven Elemente in jenen Situationen zu würdigen, die noch oder nicht mehr in Übereinstimmung mit ihrer Lehre von der Ehe sind (AL 292).

In der pastoralen Begleitung der wiederverheirateten Geschiedenen geht es um ein vertieftes Verständnis zwischen der Eucharistie und dem Ehesakrament und um die Kohärenz zwischen diesen

14 Vgl. Rocco Buttiglione, Przyjacielska odpowiedź krytykom Amoris laetitia (Kraków: Wydawnictwo WAM, 2018), 166-167. 
Sakramenten. Die Schlüsselformulierung in diesem oben zitierten Abschnitt des päpstlichen Dokuments betrifft die Widerspieglung der Einheit zwischen Christus und der Kirche in der christlichen Ehe und diese soll man, so Mroczkowski, aus der Sicht von Sacramentum caritatis von Benedikt XVI. und der darin betonten Verbindung mit der Liebe, die in beiden Sakramenten zum Ausdruck kommt, verstehen. Mangelnde Wahrnehmung des Zusammenhanges zwischen der idealen Liebe Gottes in der Eucharistie und der menschlichen, von der Begierde belasteten, ehelichen Liebe, ist laut Mroczkowski Grund der falschen Interpretationen von AL. Die Liebe zwischen Mann und Frau setzt die Zeitlichkeit der menschlichen Geschichte, die Begehrlichkeit des menschlichen Leibes und die Flüchtigkeit der zwischenmenschlichen Beziehungen voraus. Das innere Leben Gottes, ausgedrückt in der Eucharistie, begegnet der mit Leiblichkeit und Geschichtlichkeit gekennzeichneten Liebe. Daher spielen die Sakramente für die Begleitung der Ehepaare mit ihrer verletzbaren Liebe eine unentbehrliche Rolle, denn in ihnen vereinen sich das Menschliche und das Göttliche. Es beginnt auf der leiblichen Ebene, und der Leib wird in das Verständnis des Sakramentes eingeschlossen. Das so gesehene Verständnis des Sakramentes ist für das christliche Eheleben grundlegend. Nach Mroczkowski sehen einige Kommentare von AL bei Franziskus den Abschied von der metaphysischen Sicht des Ehesakramentes. Es geht dabei nicht um die Betonung der personalistischen Perspektive der Eucharistie und des Ehesakramentes, sondern um die Versuchung, die sakramentale Dimension mit der symbolischen gleichzustellen. Bei der gegenwärtigen Vielfalt der Auslegungen des Symbols müssen sich die Theologen der Bedeutung dieses Begriffs für die Sakramentenlehre bewusst sein. Soweit die Idee des Sakramentes ein symbolisches Verständnis der Welt voraussetzt, beschränkt sie sich nicht auf eine rein funktions-sachliche Bedeutung, ohne dem Menschen die Öffnung auf die geistige Dimension zu ermöglichen. ${ }^{15}$

Diese geistige Dimension des Ehesakramentes besteht, laut Mroczkowski, darin, dass der Mensch in die Geschichte des sich ihm hingebenden Gottes eingeschlossen wird und daher in keiner seiner

15 Vgl. Mroczkowski, Etos miłosierdzia a wierność matżeńska, 17-19. Mehr zur Bedeutung des sakramentalen Zeichens bei der Ehe siehe: Grzegorz Barth, 'Amoris laetitia - ciągłość czy zerwanie?: kwestie hermeneutyczne’, in Towarzyszyć matżeństwu i rodzinie: inspiracje adhortacji apostolskiej Amoris laetitia dla duszpasterstwa rodzin, ed. Jacek Goleń (Lublin: Wydawnictwo KUL, 2017), 25-50, hier 41-42. 
Lebenssituationen, auch im Eheleben, die Treue Gottes ausgelassen bleiben darf. Schließlich schützt Gott davor, dass die im Ehesakrament verankerte Unauflöslichkeit auch in Krisensituationen nicht in die Leere der Sinnlosigkeit und in die Furcht der Verzweiflung verwandelt wird. In der Ehekrise darf die Tapferkeit weder in der Idealisierung des Heroismus bestehen noch auf die Last der Verantwortung für die Gabe der göttlichen Liebe verzichten. Der christlichen Tapferkeit im sakramentalen Eheleben soll das Eheversprechen zur Unauflöslichkeit „bis der Tod uns scheidet" helfen. Die einen verstehen es als Verpflichtung, die letztendlich von der Kontemplation der Liebe Gottes kommt, die anderen als Ansporn zur Selbstüberwindung und der Überwindung eigener Schwächen. ${ }^{16}$

Bei der Verwirklichung der Unauflöslichkeit helfen den christlichen Eheleuten die Taufe und die Eucharistie. Im Sakrament der Taufe wird der Christ dauerhaft aufgerufen, gegen die Sünde zu kämpfen. Durch die Eucharistie nehmen die Christen an dem auferstandenen Leib Christi und der ekklesialen Kommunion teil, was bewirkt, dass die christlichen Eheleute ihrer Identität desto mehr entsprechen, je mehr sie an der eucharistischen Gemeinschaft der Kirche teilnehmen. Vor diesem Hintergrund wird das Wesen der Unauflöslichkeit und der Unzerstörbarkeit des Ehebundes verständlich, dessen Grundlage man in der untrennbaren Vereinigung zwischen Christus und der Kirche erblickt. Auch wenn eine begrenzte oder unbegrenzte Auflösung auf der Ebene des Leibes und der Lebensgemeinschaft möglich ist (Trennung von Bett und Tisch), ist eine zweite Eheschließung unmöglich, weil die erste Ehe dauerhaft untrennbar ist. Der unauflösliche und das ganze Leben dauernde Ehebund hat gewissermaßen den Charakter (res et sacramentum), der bei der Taufe, der Firmung und dem Weihesakrament ausgedrückt wird. Laut Mroczkowski kann man daher mit der Möglichkeit der Kommunion für die wiederverheirateten Geschiedenen nicht einverstanden sein. ${ }^{17}$

Diese knapp dargestellten Auffassungen I. Mroczkowskis müssen kritisch hinterfragt werden: Sie bedienen sich einer eigenartigen Argumentation, die vor allem die Aussagen von AL zu wenig wahrnimmt. Im päpstlichen Schreiben wird mehrmals der unauflösliche Charakter der sakramentalen Ehen betont. Es ist darin auch von einer

16 Vgl. Mroczkowski. Etos miłosierdzia a wierność matżeńska, 23-24.

17 Vgl. ebd., 25-27. 
Möglichkeit der eucharistischen Kommunion für solche wiederverheirateten Geschiedenen, die zwar in einer objektiven irregulären Situation, aber nicht im Zustand der Todsünde leben und die heiligmachende Gnade nicht verloren haben, die Rede (vgl. AL 301). Besonders relevant ist in diesem Zusammenhang die Feststellung von AL:

Aufgrund der Bedingtheiten oder mildernden Faktoren ist es möglich, dass man mitten in einer objektiven Situation der Sünde - die nicht subjektiv schuldhaft ist oder es zumindest nicht völlig ist - in der Gnade Gottes leben kann, dass man lieben und dass man auch im Leben der Gnade und der Liebe wachsen kann, wenn man dazu die Hilfe der Kirche bekommt (AL 305).

Es ist schwierig zu behaupten, dass die Möglichkeit der eucharistischen Kommunion in solchen Fällen zugleich eine Hinterfragung des unauflöslichen Charakters der sakramentalen Ehe bedeuten würde.

Andere Aspekte nimmt Marian Machinek im Hinblick auf AL im Fokus. Für ihn ist die Begründung der Zulassung zum Sakrament der Versöhnung und der Eucharistie der wiederverheirateten Geschiedenen, die zwar im Zustand der Sünde leben, aber die heiligmachende Gnade nicht verloren haben, besonders problematisch. Auch die Feststellung über die gefestigte Verbindung mit Kindern aus dieser neuen Verbindung, gewisser Treue, großherziger Hingabe, christlichen Engagements bei den irregulären Verbindungen lässt die Frage nach der Unauflöslichkeit der Ehe stellen. Machinek hat dabei ernsthafte Bedenken, ob diese Ansicht der Probleme die Hinterfragung der ontologischen Unauflöslichkeit der Ehe bedeutet oder sie dadurch beiseitegeschoben wird. ${ }^{18}$

Machinek verweist in seiner Argumentation auf den öffentlichen Charakter der Sakramente. Weder Eucharistie noch Ehe sind private Angelegenheiten, sondern haben einen öffentlichen, gemeinschaftlichen und gesellschaftlichen Charakter. Eine zwischen den Getauften geschlossene Ehe ist ein Sakrament und bildet eine übernatürliche Wirklichkeit. Daher betont die Kirche, dass das Sakrament der Eucharistie Personen nicht empfangen sollen, die in einem objektiven Zustand leben, dem die Regeln des christlichen Lebens widersprechen. Wenn man eine Gruppe von Gläubigen, die nach der Scheidung

18 Vgl. Machinek, 'Die Diskussion über die Unauflöslichkeit der Ehe’, 129. 
in einer nichtsakramentalen Partnerschaft leben, ohne die Gültigkeit der ersten Ehe zu berücksichtigen, zum Empfang der Eucharistie zulassen würde, würde es faktisch eine Akzeptanz und Anerkennung einer zweiten Ehe der Getauften nach der Scheidung bedeuten. Auf diese Weise würde die Kirche die wiederverheirateten Geschiedenen zur Eucharistie zulassen und dadurch das Leben auf eheliche Weise mit einer Person, die nicht ein Ehepartner ist, legalisieren. ${ }^{19}$

Auf die Begründung der Kohärenz zwischen den Sakramenten beruft sich bei der Interpretation von AL auch Marian Pokrywka. Er argumentiert, dass man die einzelnen Sakramente nicht voneinander unabhängig und getrennt, sondern integral behandeln soll. Der Empfang eines Sakramentes verlangt eine richtige Beziehung zu allen anderen, weil man denselben Christus nicht in einem Sakrament annehmen, in einem anderen aber ablehnen kann. Aus dieser Sicht sollen die Probleme der Ehescheidung und der zivilen Wiederverheiratung betrachtet werden. Die Kirche hat nach ihrer bisherigen Lehre die wiederverheirateten Geschiedenen zu den Sakramenten nicht zugelassen. Es war keine Kirchenstrafe, sondern Menschen in solchen Situationen, so Pokrywka, haben sich selbst ausgeschlossen, ihr Lebensstand war im Widerspruch zum christlichen Eheverständnis und daher ein Hindernis für einen fruchtbaren Empfang des Sakramentes, solange dieser Zustand dauerte. Leben in einer nichtsakramentalen Partnerschaft - verbunden mit Geschlechtsverkehr - ist ein objektiver Zustand der Todsünde und macht ohne Bekehrung eine Lossprechung und einen Kommunionempfang unmöglich. Personen, die einen gültigen und unauflöslichen Ehebund geschlossen und dann zivil geschieden in einer zweiten Partnerschaft leben, erfüllen diese Voraussetzungen nicht. Ausschlaggebend bei der Nichtzulassung der wiederverheirateten Geschiedenen zu den Sakramenten soll nicht der persönliche Zustand der Gnade, sondern ein offensichtlicher Widerspruch auf der öffentlichen und sakramentalen Ebene gegenüber der Eucharistie sein. Entscheidend ist der objektive Widerspruch auf der Ebene der sakramentalen Kohärenz und nicht die subjektive Schuld der Personen. ${ }^{20}$

Pokrywka betont die ontologische Bedeutung der Unauflöslichkeit der Ehe und ihren Zusammenhang mit der Eucharistie. Durch

19 Vgl. Machinek, 'Logika inkluzji papieża Franciszka', 205.

20 Vgl. Pokrywka, 'Teologicznomoralne implikacje adhortacji Amoris laetitia', 91-93. 
das Kreuzesopfer Christi, das in der Kirche ständig in der Eucharistie gegenwärtig ist, wurden Mann und Frau in der Würde des biblischen „Anfangs" vereint und von der „Verhärtung des Herzens“, dass heißt ihre Unfähigkeit der gegenseitigen Hingabe, auf immer befreit. Erst in dieser Perspektive kann man richtig die wahre Natur der Unauflöslichkeit der Ehe verstehen, die keine moralische oder rechtliche Pflicht ist, sondern eine Gabe, die ontologisch die Personen der Ehegatten formt, wenn sie zu einem Band verbunden werden, das ein reales Zeichen der Einheit zwischen Christus und Kirche ist. In der Ehe handelt göttliches Wirken, das Personen vereint. Im ehelichen Versprechen ist ein Geschehen vorhanden, das das bewirkt und überschreitet, was die Ehepartner tun, und sie in einem Mysterium verwurzelt. Das Wesen dieser Verwurzelung ist die Unauflöslichkeit. Das, was Gott gibt, bleibt auf ewig, er ruft seine Gaben nie zurück. So ist die Ehe ontologisch unauflöslich und weder der Wille des Menschen noch die Willkür der einzelnen Ehen noch die Entscheidungen der Institutionen verfügen über sie. ${ }^{21}$

Die theologische Kritik der Ausführungen von Machinek und Pokrywka kann auf den Überlegungen von Kardinal Walter Kasper aufgebaut werden. Mit Recht betont er, dass AL die Worte Jesu zu Ehebruch und Ehescheidung (Mt 5,31-32; 19,3-12) nicht aufhebt, sondern sie anwendet. Daher ist die Aussage von Johannes Paul II. aus Familiaris consortio weiterhin gültig. Es geht um die Position, dass eine zivile Ehe bei Fortbestand einer ersten sakramentalen Ehe in objektivem Widerspruch zum unauflöslichen sakramentalen Bund der ersten Ehe steht (vgl. FC 84). Diese unverrückbare Lehre der katholischen Kirche wird in AL nicht bestritten, sondern bekräftigt. Aus diesem Grundsatz folgt, dass eine zivile Ehe unter Fortbestand der ersten sakramentalen Ehe keine sakramentale Ehe sein kann und eine solche nichtsakramentale Ehe nicht durch eine liturgische Segenshandlung verbunden werden darf, weil es die Unauflöslichkeit der Ehe ins Zwielicht rücken würde. Christen, die in solchen irregulären Situationen leben, sind nicht exkommuniziert, sondern eingeladen, sich als lebendige Mitglieder am kirchlichen Leben zu beteiligen. Wenn AL in einzelnen Fällen unter bestimmten Voraussetzungen solche Paare zu den Sakramenten der Versöhnung und der Eucharistie zulässt, ist als nächster Schritt laut Familiaris consortio anzusehen, wonach zur Absolution

21 Vgl. ebd., 85-86. 
und Kommunion wiederverheiratete Geschiedene zugelassen wurden, die sich dafür entschieden haben, „völlig enthaltsam zu leben, d.h. sich der Akte zu enthalten, welche Eheleuten vorbehalten sind" (FC 84). Kasper sieht diese Klausel als Zugeständnis, denn Enthaltsamkeit gehört zum Intimbereich und hebt den objektiven Widerspruch zwischen dem fortbestehenden Ehebund der ersten sakramentalen Ehe und der zivilen nichtsakramentalen Ehe nicht auf. Diese Klausel zeigt, dass es in der konkreten Ausgestaltung der praktischen pastoralen Konsequenzen des dogmatischen Prinzips einen Spielraum gibt. ${ }^{22}$

Laut Kardinal Francesco Coccopalmerio wird die Lehre von der Unauflöslichkeit der Ehe bei der Möglichkeit des Zugangs zu den Sakramenten der wiederverheirateten Geschiedenen, die AL unter besonderen Bedingungen nicht ausschließt, in keiner Weise in Frage gestellt, sondern bewahrt. Es wird anerkannt, dass diese Christen in irregulären Verbindungen leben und diese Lebensweise objektive Umstände der Todsünde bildet. Wenn sie jedoch in einzelnen Fällen zu den Sakramenten zugelassen werden, wird dabei auch die Lehre von der Reue, die eine Veränderung der Lebensführung nach sich zieht, als unentbehrliche Voraussetzung der Lossprechung bewahrt. Gläubige in diesen Situationen sind sich einerseits bewusst und davon überzeugt, dass die Situation, in der sie leben, objektiv sündhaft ist, und andererseits haben sie den Vorsatz, das Leben zu ändern, auch wenn sie nicht imstande sind, ihn zu verwirklichen. Der Vorsatz ist die theologische Grundlage zur Sündenvergebung und des Zugangs zur Eucharistie, und in diesem Falle verbunden mit der Unmöglichkeit der sofortigen Änderung der sündhaften Situation aufgrund schwerwiegender Gründe. ${ }^{23}$

Amoris laetitia behandelt dieses Problem aus dem Gesichtspunkt der mildernden Umstände und stellt fest:

Die Kirche ist im Besitz einer soliden Reflexion über die mildernden Bedingungen und Umstände. Daher ist es nicht mehr möglich zu behaupten, dass alle, die in irgendeiner sogenannten ,irregulären' Situation leben, sich in einem Zustand der Todsünde befinden und die heiligmachende Gnade verloren haben (AL 301).

22 Vgl. Kasper, 'Amoris laetitia: Bruch oder Aufbruch?', 727-728.

23 Vgl. Francesco Coccopalmerio, Radość miłości: przewodnik po ósmym rozdziale adhortacji Amoris laetitia papieża Franciszka (Dodatek specjalny do „Tygodnika Powszechnego", Kraków, 2017), 27. 


\section{Schlussüberlegungen}

Die meisten Diskussionen zu Amoris laetitia und ihre Stellungnahmen zum Verständnis der Ehe in der Gegenwart betreffen die Frage nach der Behandlung der wiederverheirateten Geschiedenen in der Pastoral und ihren Zugang zu den Sakramenten. Diese Probleme sind mit der Frage nach der Unauflöslichkeit der Ehe untrennbar verbunden. In den Beiträgen und Abhandlungen ausgewählter polnischer Moraltheologen wird die Unauflöslichkeit der Ehe nach der Entscheidung von AL als in Gefahr angesehen. In ihnen wird der Vorwurf erhoben, dass der normative Charakter der Unauflöslichkeit der Ehe zu wenig betont bzw. in Frage gestellt wird und die Kohärenz zwischen der Eucharistie und dem Ehesakrament geschwächt bzw. übersehen wird. Im vorliegenden Beitrag wurden beide Argumente diskutiert und erläutert, dass weder Begriffe noch die ungenügende normative Sprache noch der in bestimmten Situationen ermöglichte Zugang der wiederverheirateten Geschiedenen zu den Sakramenten den Grundsatz der christlichen Ehelehre zu ihrer Unauflöslichkeit in Frage stellen.

Die in AL verwendeten Begriffe wie „Ideal“, „Barmherzigkeit“, „Geschenk" beabsichtigen in keiner Weise die gültigen Normen im Bereich der Ehe- und Familienethik in Frage zu stellen, sondern wirksamer, glaubwürdiger, angemessener und überzeugender das Evangelium in der Pastoral zu verkündigen und umsetzen zu lassen. Eine Möglichkeit des Sakramentsempfangs für die Gläubigen in den sogenannten irregulären Situationen, die für viele polnischen Moraltheologen bedenklich ist, bedeutet in keiner Weise die Hinterfragung der Unauflöslichkeit der Ehe sowie die Legalisierung der nichtsakramentalen Partnerschaften. Vielmehr liegt das Ziel darin, in der Situation, in der subjektive Schuld schwer zuzurechnen ist, der Gnade und der Liebe Gottes den Weg zu ermöglichen. Es kann auch eine Hilfe bedeuten, die sowohl vom Sakrament der Versöhnung, in dem man die Barmherzigkeit Gottes erfährt, als auch vom Sakrament der Eucharistie, die keine Belohnung für die Vollkommenen, sondern ein Heilmittel und eine Nahrung für die Schwachen ist, ausgeht.

Die besprochenen Bedenken mancher polnischen Moraltheologen hinsichtlich der Unauflöslichkeit der Ehe nach AL muss man auf dem Hintergrund der lokalen Situation sehen, aus der die Autoren kommen. Das erste Element dieses Kontextes liegt auf der pastoralen Ebene. Es handelt sich um die Tatsache, dass in Polen viele Gläubige das 
Sakrament der Buße und der Versöhnung praktizieren und die Beichtväter eine Klarheit und anzuwendende praktische Lösungen hinsichtlich der Lossprechung von den Theologen und den kirchlichen Dokumente erwarten. Der zweite Grund liegt auf der theologischen Ebene. Es ist die Hochschätzung der Lehre von Johannes Paul II., die man durch die Lehre von AL gefährdet sieht - was jedoch gar nicht stimmt, wenn man Familiaris consortio und AL tiefer reflektiert und sie vergleicht.

Wydziat Teologiczny Uniwersytetu Opolskiego $P L-45342$ Opole E-mail:kglombik@uni.opole.pl 